\title{
Shared Access Satellite-Terrestrial Reconffiurable Backhaul Network Enabled by Smart Antennas at MmWave Band
}

Xavier Artiga, Ana Pérez-Neira, Jorge Baranda, Eva Lagunas, Symeon Chatzinotas, Rudolf Zetik, Przemyslaw Gorski, Konstantinos Ntougias, David Pérez, and Georgios Ziaragkas

\begin{abstract}
5G traffic expectations require not only the appropriate access infrastructure, but also the corresponding backhaul infrastructure to ensure well-balanced network scaling. Optical fiber and terrestrial wireless backhaul will hardly meet 100 percent coverage, and satellite must be considered within the $5 \mathrm{G}$ infrastructure to boost ubiquitous and reliable network utilization. This work presents the main outcomes of the SANSA project, which proposes a novel solution that overcomes the limitations of the traditional fixed backhaul. It is based on a dynamic integrated satellite-terrestrial backhaul network operating on the mmWave band. Its key principles are seamless integration of the satellite segment into terrestrial backhaul networks, a terrestrial wireless network capable of reconfiguring its topology according to traffic demands, and aggressive frequency reuse within the terrestrial segment and between terrestrial and satellite segments. The two technological enablers of SANSA are smart antenna techniques at $\mathrm{mmW}$ ave and software defined intelligent hybrid network management. This article introduces these 5G enablers, which permit satellite communications to play a key role in different $5 \mathrm{G}$ use cases, from the early deployment of 5G services in sparse scenarios to enhanced mobile broadband in denser scenarios.
\end{abstract}

\section{INTRODUCTION}

5G targets orders of magnitude improvement in performance metrics such as capacity, latency reliability, and availability. Furthermore, 5G pursues ubiquitous coverage, ensuring service continuity from dense urban scenarios to sparsely populated areas or in transit between them [1]. Clearly, there is no use case requiring all these metrics at the same time, or a single technology capable of providing them. High-capacity small cells combined with optical fiber backhaul are positioned to offer the best performance in the hottest spots of our cities. However, as we move away from the city centers, optical fiber deployments are scarce and expensive, so alternative technologies such as wireless or satellite backhaul will play a major role. Indeed, satellite communications have been identified as the most cost-affordable technology to meet the 5G coverage requirements [2].

For the sake of efficiency, 5G also represents a shift of paradigm in the design and management of telecommunication networks, from fixed to flexible solutions capable of adapting to different service needs. However, current wireless backhaul networks above $6 \mathrm{GHz}$ consist of high directional links forming fixed topologies, which are designed through extensive radio planning campaigns. Their capabilities to deal with failure or congestion events are very limited and constrained to the activation of redundant equipment or to the change of the flow direction in the case of ring topologies. Therefore, they cannot fully adapt the network topology to changes in traffic profiles, automatically react to link failures, or easily include new nodes in the network, which are essential features in future 5G dense deployments.

Current wireless backhaul solutions are also affected by inflexible and too conservative spectrum management policies, which result in inefficient spectrum utilization, especially considering the identified spectrum scarcity for meeting $5 \mathrm{G}$ demands. Besides, current policies also prevent in general the spectrum coexistence between different services such as fixed satellite service (FSS) and microwave backhaul links, although some exceptions are found such as the extended Ka-band (17.7-19.7 GHz) [3]. However, even in this case, interference mitigation techniques are needed to protect satellite receivers from powerful terrestrial transmitters.

In order to overcome the aforementioned limitations of traditional backhaul solutions, the H2020 SANSA project [4] proposes a spectrum-efficient self-organizing integrated terrestrial-satellite backhaul network operating at the extended Ka-band. The key enablers of the SANSA solution are a novel hybrid network management scheme that makes the most efficient use of all network resources coming from the terrestrial and satellite segments, and smart antennas providing network topology reconfiguration and spatial interference mitigation. Satellite backhaul of isolated cells is already a reality, but the seamless integration of the satellite in terrestrial backhaul networks has received very limited and recent attention [5]. Similarly, the consideration of topology reconfiguration in wireless backhaul 
networks has been discussed recently in the context of small cells [6]. However, the integration of the satellite in a dynamically reconfigurable backhaul network, for the efficient use of all network resources given the traffic requirements, is a unique proposition from SANSA.

This work presents, for the first time, the SANSA solution as a whole, from the main concept to the design and evaluation of its main enablers, concluding with their experimental validation. The novel contributions herein are:

- The joint evaluation of the topology management algorithm and the dynamic routing solution, which compound the core function of the hybrid management scheme

- The experimental validation of this scheme

- The over-the-air demonstration of the spectrum sharing between terrestrial and satellite links thanks to hybrid analog-digital arrays In addition, we also present novel results on multi-antenna techniques enabling spectrum sharing, although the details on the techniques have been introduced in a previous publication [7].

The article is organized as follows. The following section introduces the SANSA concept and architecture; then we present the insights of the novel hybrid management scheme. Following that, we detail the spectrum sharing enabling techniques and then explain the experimental platform for the validation of the main SANSA enablers. Finally, we discuss follow-up research opportunities and conclude the work.

\section{SANSA OVERVIEW}

The SANSA concept aims to improve traditional wireless backhaul networks in terms of coverage, reliability, capacity, latency, energy, and spectrum efficiency. The solution is based on the seamless integration of the satellite segment in a self-organizing terrestrial wireless network capable of adapting its network topology to traffic needs, and on the aggressive frequency reuse among terrestrial backhaul links and among terrestrial and satellite segments. This solution is enabled mainly by smart antennas deployed at the terrestrial nodes and by a novel hybrid network management scheme. As shown in Fig. 1, this scheme is based on two main entities, namely the Hybrid Network Manager (HNM) and the intelligent Backhaul Node (iBN). The HNM is the central element of the SANSA network, which collects information and event alerts (e.g., link failures or congestion) from all network resources, defines traffic policies, and reconfigures the topology of the transport backhaul network according to its current state. It is composed of an event manager, which deals with the received information; a topology manager, which calculates alternative topologies to solve network events; a configuration manager, which sends configuration instructions to the distributed iBNs; and a radio resource manager. The latter is in charge of handling interference mitigation measures and assisting the topology manager since different network topologies imply different interference landscapes. The iBNs extend traditional backhaul nodes, including new blocks and interfaces, for flexible management and seamless integration of its equipped terrestrial modems, smart antennas, and satellite modems in the case of hybrid nodes. The iBNs

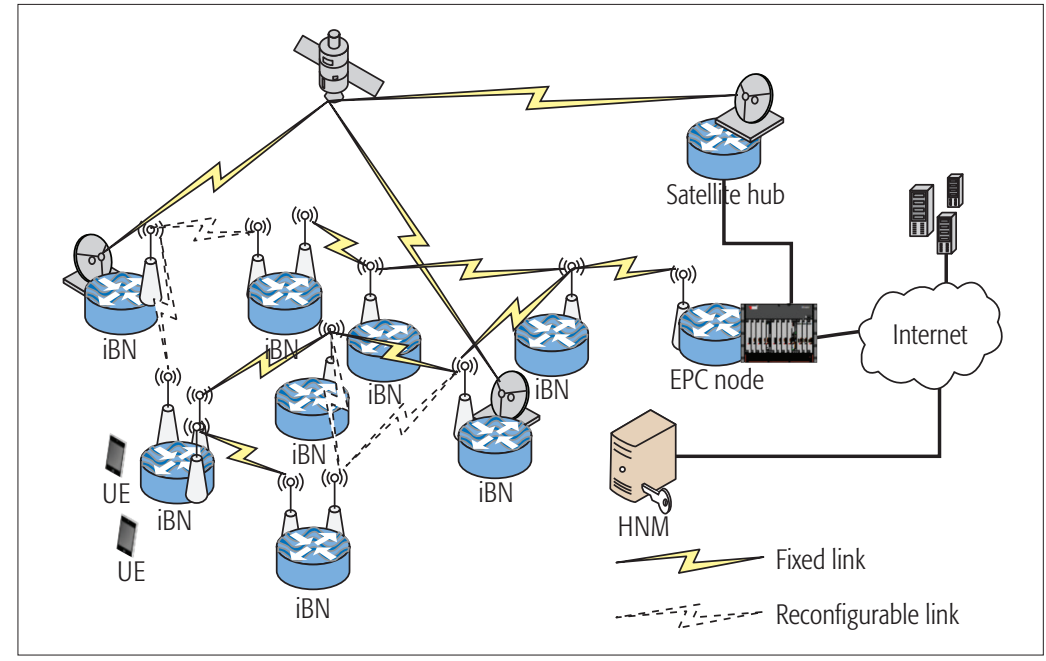

FIGURE 1. SANSA system environment.

are thus responsible for reconfiguring both hardware (smart antennas) and software (traffic offloading) according to the guidelines sent by the configuration manager of the HNM. In addition, they execute distributed routing, load balancing, and traffic classification functions for the efficient use of all network resources.

The SANSA architecture is a suitable solution for many fifth generation (5G) related use cases. In particular, the integration of the satellite facilitates early 5G service deployments in sparse scenarios and ensures service continuity from dense to sparse scenarios. Jointly with the bench of functionalities provided by the $\mathrm{HNM} / \mathrm{IBNs}$, the satellite also contributes to provide a flexible solution for efficiently improving enhanced mobile broadband (eMBB) services in denser scenarios in terms of capacity, latency, and reliability. Although not addressed here due to space constraints, SANSA also developed energy management [8] and offline caching functions [9] for the iBNs. Therefore, the SANSA solution will contribute to energy consumption reductions in dense small cell deployments by smartly setting small cells with low traffic demands in sleep mode. Finally, SANSA will contribute to important bandwidth savings in backhaul networks thanks to a hybrid terrestrial-satellite caching scheme. Remarkably, the satellite plays a major role in this scheme, providing efficient placement of content in edge caches due to their wide coverage and inherent multicast capabilities.

\section{Hybrid Network Management}

This section details and evaluates the solution for the dynamic and efficient reconfiguration of the network, which is the core functionality of the proposed hybrid network management scheme. Therefore, it focuses on the topology management and the designed distributed routing, illustrating the main point of interaction between the HNM and the iBNs. Further details on the internals of all HNM/iBN functionalities as well as extensive simulation campaigns can be found in $[8,9]$.

Within the HNM, the topology manager is responsible for determining the terrestrial network topology by reconfiguring the smart anten- 


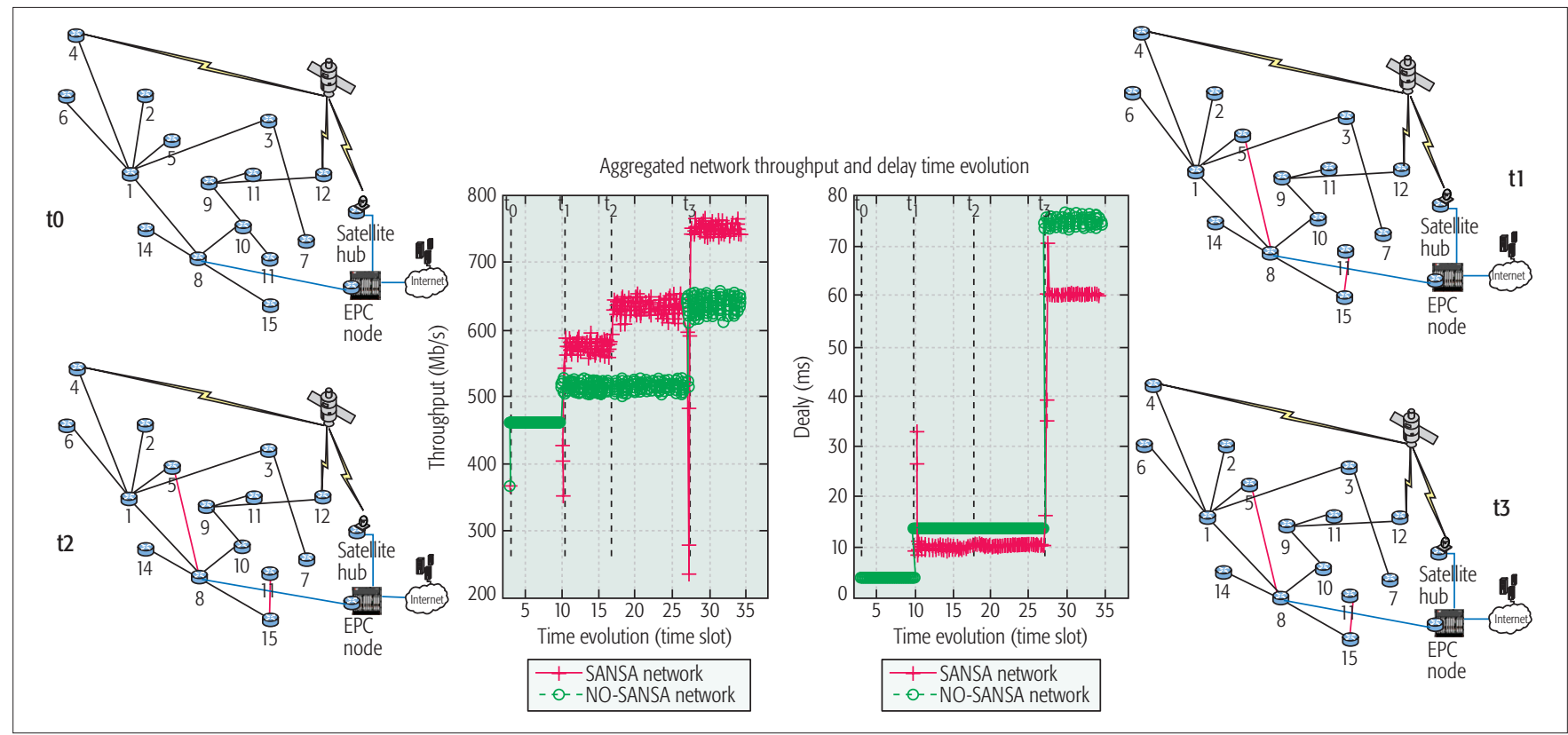

FIGURE 2. Aggregated network throughput and delay over time when evaluating the reference scenario using the SANSA network with respect to a non-SANSA network.

nas at the iBNs to change their link directions. The final objective is to improve the use of network resources for given traffic conditions. When a network event reported by the iBNs arrives at the HNM (i.e., a link failure or congestion), the topology manager calculates a possible set of candidate topologies to solve the event, based on the current network state. Then the candidate topologies are scored, calculating a set of indicators on each of them. The network operator can configure the number and weight of indicators, which include network similarity, power consumption, delay, and bandwidth, among others. In addition to the topology change, the HNM can also decide to change the traffic policy (i.e., traffic balance usage between terrestrial and satellite resources) to react in the face of congestion episodes.

The HNM communicates the new topology and the changes in the traffic policy to the iBNs through the configuration manager. Based on this information, the iBNs apply the corresponding actions in the configuration of their terrestrial and satellite modems and smart antennas.

In order to be able to exploit this dynamicity in the network topology configuration, iBNs use the designed Backpressure for Multi-Radio (BP$\mathrm{MR}$ ) routing protocol [10]. Contrary to state-ofthe-art (SoA) approaches, such as the ones based on shortest path (multiprotocol label switching [MPLS], Internet Engineering Task Force [IETF] RFC 5921), BP-MR allows seamless integration of satellite and terrestrial resources while exploiting the path redundancy offered by the envisioned hybrid backhauls. BP-MR is based on the Lyapunov drift-plus penalty approach proposed by Neely [11], where routing decisions are made in a distributed fashion at each iBN, combining queue backlog information (Lyapunov drift) with geographic information (penalty component). The relative importance of each component is adjusted dynamically through a parameter that finds the best trade-off between congestion avoidance and path length. Essentially, BP-MR performs routing decisions following a two-stage process. First, it classifies data packets in a per-interface queue system according to their final destination. Second, it employs geographic and congestion information to compute the best possible next hop from all possible forwarding options. This decision is made for each multi-radio backhaul node at each transmission instant. Congestion information is obtained through the periodical exchange of control packets, called HELLO packets, between neighboring iBNs. The granularity of the routing decision is also configurable, and it can be done on a per-packet or per-flow basis. The per-packet approach provides better usage of the network resources; however, it may spread packets belonging to the same flow over different paths so that they are received out of order at the destination, creating problems for a TCP receiver. In such cases, the per-flow basis decision can overcome the packet-reordering problem. Identifying a flow as an origin to destination stream of a transport layer connection between two end-hosts, each node maintains active per-flow state information, mapping the packets of a flow to its determined path, calculated the first time the node sees the flow. Hence, the iBN has the flexibility to route dynamically a new flow while still circumventing congested routes. This state information is eliminated at each iBN after the flow is not present in the network for a configurable period of time.

Next, we present the joint operation of the topology manager of the HNM and the BP-MR routing protocol running at the $i B N s$ in a reference scenario based on a real network deployment close to Helsinki, as depicted in Fig. 2. This scenario counts 15 backhaul nodes, 2 of them having terrestrial and satellite connection capabilities. The link rate of such links has been derived from an interference analysis and the characteristics of commercial equipment used in terrestrial backhaul deployments. Simulation results in Fig. 2 show the time evolution of the achieved 
throughput and packet delay when comparing the SANSA approach vs. a non-SANSA benchmark solution. This last is characterized by a terrestrial segment and a satellite segment with a fixed topology (the one depicted as t0 in Fig. 2) using an SoA routing solution.

Initially, at instant t0, both approaches, SANSA and non-SANSA, perform equally since there are enough network resources to serve the requested traffic demanded by iBNs, labeled 1, 2, 3, 9, 10, 11, 14, and 15. At instant t1, iBNs start reporting congestion to the HNM due to the increase of demanded throughput (activation of iBNs 6 and 13). The HNM reacts to this congestion episode by changing the network topology. In this evaluation, the most relevant criteria to determine the new topology is the network similarity, that is, to constrain the number of topological changes with respect to the current/baseline topology. The introduced changes in network topology at $\mathrm{t} 1$ are depicted with red lines in Fig. 2. Thanks to these changes, the SANSA-network is able to satisfy the requested traffic. At $t 2$, there is an additional increase of traffic requested by iBN 7. Interestingly, the lack of congestion reports from the iBNs produces no activation by the topology manager module embedded in the HNM. Requested traffic can be served thanks to the capabilities of BP-MR to exploit available network resources. On the other hand, we can see that the non-SANSA network has entered saturation, and it is not able to cope with the throughput increase. Finally, at instant $\mathrm{t} 3$ there is an additional demand of traffic downloads in other IBNs (iBNs 4 and 12). In this case, the HNM determines a change in the traffic policy, offloading a total of 20 percent of the traffic directed to each iBN to enter the backhaul network through the iBN equipped with satellite modems. This offloading has also been simulated for the non-SANSA scenario, highlighting the additional gains introduced by the BP-MR routing protocol. As depicted in Fig. 2, the SANSA network offers around a 50 percent throughput improvement with respect to the non-SANSA network while being able to serve all the traffic in the case of more network usage (i.e., after $\mathrm{t} 3$ ). Even providing the non-SANSA network with satellite resources, the SANSA network still offers a 15 percent throughput improvement thanks to the BP-MR routing protocol. With respect to the latency, Fig. 2 shows an improvement of 23 percent, which goes up to 35 percent when considering only terrestrial traffic.

\section{SPECTRUM SHARING}

This section describes the techniques integrating the radio resource manager module of the HNM, which assists the topology manager in order to contain the effects of interfering signals, allowing the sharing of the spectral resources in an efficient manner. Two technological enablers are proposed: smart radio resource management (RRM) techniques and multi-antenna techniques such as beamforming and null-steering. The results are presented in terms of network spectral efficiency (NSE), defined here as the ratio between the sum-rate across the whole network and the total available bandwidth [12]. As a difference from traditional link spectral efficiency, which only considers the used bandwidth, here we consider the
The RRM gain arises from efficiently packing a high number of wideband backhaul links into a limited

spectral band. Consequently, we initially investigate the interference problem from a simple carrier

allocation point of view.

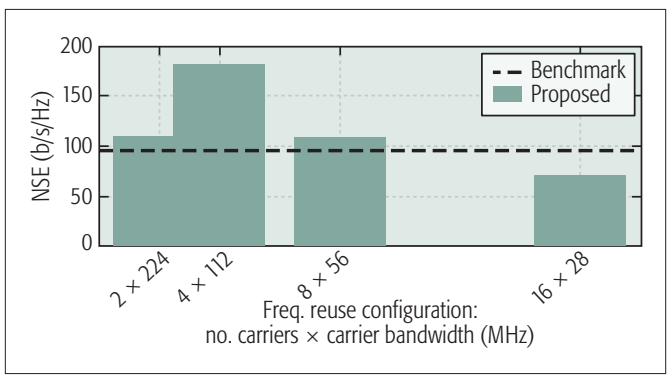

FIGURE 3. NSE of the integrated backhaul network as a function of the number of carriers used to operate the network links.

available bandwidth to capture frequency reuse effects.

\section{DYNAMIC RRM}

The RRM gain arises from efficiently packing a high number of wideband backhaul links into a limited spectral band. Consequently, we initially investigate the interference problem from a simple carrier allocation point of view. Carrier allocation has been extensively studied in terrestrial backhaul networks, but rarely considering the terrestrial and satellite spectrum sharing assumption [13]. In general, the coupling between the terrestrial carrier assignment and the satellite backhaul link rates makes the carrier allocation problem very challenging. To overcome the coupling issue, we propose a two-step suboptimal method based on the sum-link rate maximization, where we first determine the carrier allocation of the satellite system and, in a second step and assuming the resulting satellite segment allocation, we design the carrier assignment for the terrestrial segment.

As an illustrative example, let us consider the reference Helsinki scenario introduced in Fig. 2 assuming that we have a limited number of wideband carriers to operate the 14 bidirectional links of such a network. Figure 3 illustrates the NSE obtained with the proposed two-step carrier allocation algorithm for different frequency reuse configurations. As a benchmark, we consider the original pure terrestrial network featuring a configuration based on 8 carriers of $56 \mathrm{MHz}$ of bandwidth and hence a total available bandwidth of $448 \mathrm{MHz}$ (which is kept constant for all other configurations). From right to left, it can be observed that the NSE increases when the number of carriers reduces, since the proposed carrier allocation algorithm is able to efficiently manage the resulting interference among the wideband carriers. However, a saturation point is achieved when the number of carriers is too small, drastically degrading the NSE growth trend. This point corresponds to the case where the carrier allocation algorithm is no longer able to manage the resulting interference. In conclusion, the proposed method allows high frequency reuse achieving a $2 \times$ NSE gain compared to the benchmark. This 


\begin{tabular}{|c|c|c|c|c|c|}
\hline \multirow{2}{*}{ Scenario } & \multirow{2}{*}{$\begin{array}{c}\text { Benchmark (orthogonal } \\
\text { frequency reuse) } \\
\text { Network SE }\end{array}$} & \multicolumn{2}{|c|}{$\begin{array}{l}\text { Aggressive frequency reuse without interference } \\
\text { mitigation }\end{array}$} & \multicolumn{2}{|c|}{$\begin{array}{c}\text { Aggressive frequency reuse with interference } \\
\text { mitigation }\end{array}$} \\
\hline & & Network SE & Gain w.r.t. benchmark & Network SE & Gain w.r.t. benchmark \\
\hline Spectrum sharing, urban & 3.61 & 7.6 & 2.1 & 10.16 & 2.8 \\
\hline Spectrum sharing, rural & 3.61 & 8.3 & 2.3 & 12.04 & 3.3 \\
\hline
\end{tabular}

TABLE 1. Simulated Network spectral efficiency in b/s/Hz.

gain can be further increased to $2.5 \times$ when the carrier allocation is combined with power and flow control, taking into account the traffic that each link can support [14]. Higher NSE gains can be achieved without the need for additional spectrum by applying multi-antenna beamforming and precoding for further interference mitigation, as described in the following section.

\section{MuLtT-AntenNA TeCHNIOUES}

SANSA deploys antenna arrays at the backhaul nodes to enable topology reconfiguration and frequency reuse. At millimeter-wave (mmWave), a large number of antenna elements are required to combat path loss. Therefore, given the cost performance trade-off, we opt for a hybrid analog-digital (HAD) architecture, where a low-dimensional digital beamformer operates at baseband while the analog beamformer maps a reduced number of RF chains to a large number of antenna elements [7]. For further complexity reduction, we consider here a partially connected structure, in which each antenna connects to only one RF chain, resulting in analog subarrays placed side by side. In addition, each analog subarray only has phase control capabilities.

The analog and digital beamformers are optimized in order to maximize the gain in the desired direction while containing the interference levels to multiple non-intended receivers below a given threshold. The optimization proposed here is based on an alternating projection method that iteratively solves the analog and digital beamformers. The analog part is solved through a feasible point pursuit successive convex approximation (FPP-SCA) for dealing with the quadratic inequality constraints that appear due to the phase-only control; second order cone programming is used for the digital part. The interested reader can refer to [7] for further details on this technique.

This solution is evaluated in two different simulation scenarios addressing the spectrum sharing between terrestrial and satellite segment and the aggressive frequency reuse among terrestrial links, respectively. The former considers the NSE achieved by multiple satellite receivers randomly located in the vicinity of a single terrestrial link. Table 1 shows the results of Monte Carlo simulations in both rural and urban conditions. In all cases, the considered available bandwidth is 2 $\mathrm{GHz}$ of the shared Ka-band (17.7-19.7 GHz), except in the benchmark, where we considered the conventional situation in which the satellite receivers only use the satellite exclusive band (i.e., $500 \mathrm{MHz}$ ). By the simple use of a larger shared bandwidth, the NSE increases by a factor of $2 \times$, even without using HAD beamforming, whereas it goes up to a factor of $2.8 \times$ and $3.3 \times$ when applying it in the rural and urban scenarios, respectively. In conclusion, HAD permits approaching the theoretical $4 \times$ gain improvement that comes from using a shared bandwidth $4 \times$ larger.

The second scenario addresses the NSE achieved by the reference Helsinki network assuming a frequency reuse scheme of 2 (to avoid full-duplex communication) and nodes equipped with a 64-antenna array. As a benchmark, we consider the nodes equipped with a conventional directive dish antenna and the benchmark carrier allocation introduced before (i.e., 8 carriers of $56 \mathrm{MHz}$ bandwidth). As shown in Table 1, the aggressive frequency reuse (from 8 carriers to 2 ) has a strong impact on the NSE going from 330 $\mathrm{b} / \mathrm{s} / \mathrm{Hz}$ to $2.9 \mathrm{~b} / \mathrm{s} / \mathrm{Hz}$ when considering the conventional dish antenna. On the other hand, the proposed HAD solution is able to provide NSE gains up to $3.5 \times$ with respect to the benchmark, thus close to the theoretical $4 \times$ gains that would come from using 2 carriers instead of 8 . We note that NSE gains up to $9 \times$ have also been demonstrated by combining spatial multiplexing with beam and null steering in the same scenario [12] .

\section{SANSA Experimental PlatForm}

Technical research activities carried out in the SANSA project achieved the targeted technology readiness level (TRL) 4; thus, a technological validation was performed in a virtual electromagnetic environment at the facility for over-theair research and testing (FORTE) at Fraunhofer Institute for Integrated Circuits IIS. This allowed us to test the basic functionalities of the proof of concept (PoC) of the two SANSA enablers, namely a HAD antenna array and the HNM/iBN pair. Therefore, two small-scale demonstrations in realistic but well controlled scenarios were performed, as detailed next.

\section{Demonstration of Multi-Antenna Array at \\ Ka-BAnd And InterFerence Mitigation TeChnIQUeS}

The first demonstration scenario focused on the interference mitigation using a hybrid (analog-digital) beamforming antenna array PoC [15]. It operates at the extended Ka-band and consists of 64 transmitting antenna elements that are arranged in two side-by-side linear subarrays. Each single-antenna element is connected to a phase shifter and an attenuator that are controlled by 8 bits for analog beamforming. Two RF chains that drive the two subarrays were connected to a channel emulator that executed the digital baseband precoding. In this constellation, the analog antenna array and the digital channel emulator were able to perform hybrid (analog-digital) beamforming. 


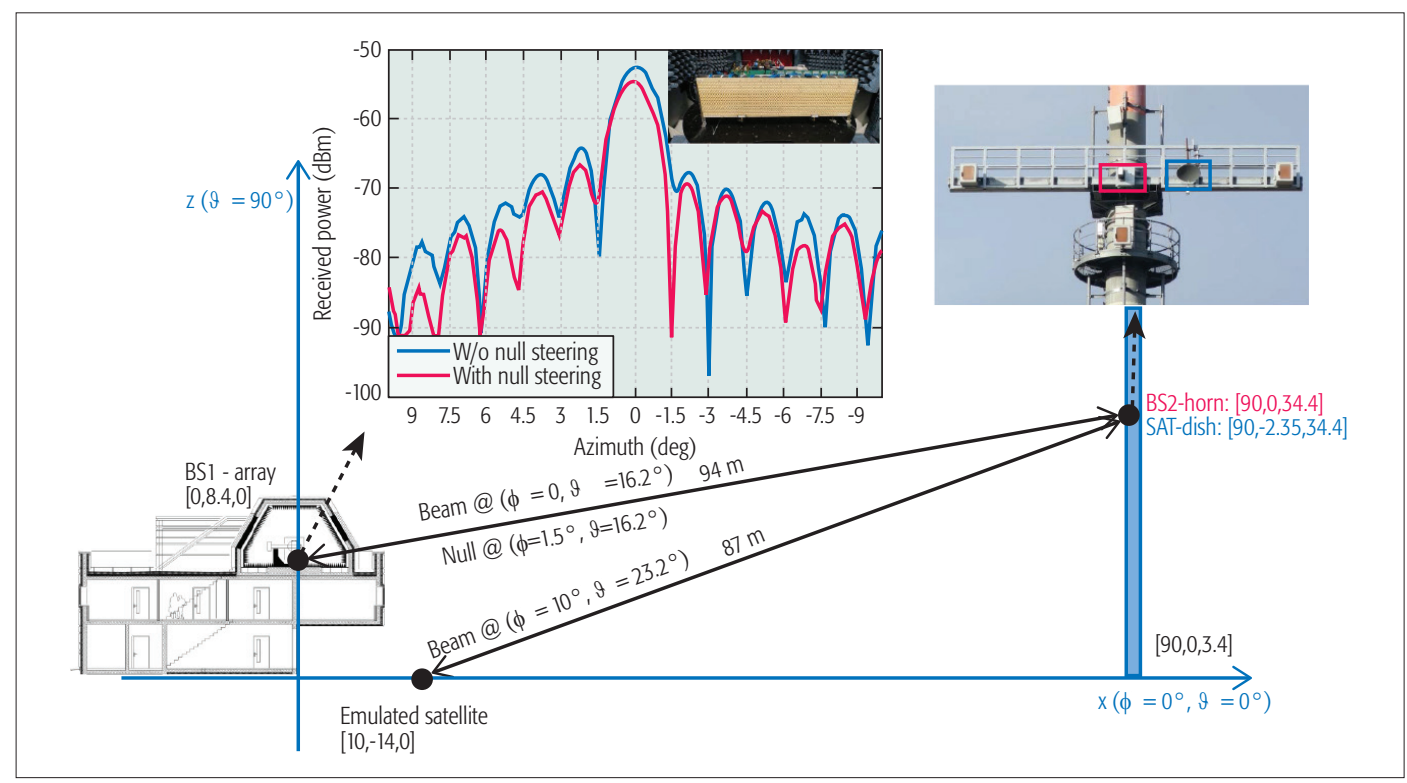

FIGURE 4. Side view of the OTA experiment with dimensions in meters. The inset graph shows the measured beam patterns of the antenna array prototype (inset), steering one beam to 0 by means of analog-only beamforming (blue) and forming one beam at 0 and one null at 1.5 by means of hybrid beamforming (red).

The inset in Fig. 4 shows the antenna array mounted at a motion emulator in an anechoic chamber, which faced a nearby antenna tower through an electromagnetic transparent window. A horn antenna was mounted on this tower for receiving the transmitted signal and evaluating radiation patterns.

The inset curves in Fig. 4 illustrate the beamforming capabilities of the hybrid antenna array. The blue line shows the measured beam pattern of the analog only antenna array steering one beam to the boresight. The red line in Fig. 4 shows the measured beam pattern of the array obtained by the hybrid beamforming. Even with only two RF chains (i.e., only two complex beamforming weights), the hybrid beamformer could properly form the beam at 0 and a deep null at 1.5. The decrease of the beam power is due to the normalization that sets the maximum power of the digital weights to one. This resulted in a reduction of the total radiated power in the case of the hybrid beamforming. Still, the comparison is realistic since it reflects that beamforming weights cannot be set above a predefined maximum level.

The hybrid beamforming was the basis for the spectrum sharing demonstration with one terrestrial and one satellite link, depicted in Fig. 4. The forward terrestrial link and the downlink of the satellite link were emulated over the air between the laboratory building and the nearby antenna tower (@ 100 m distance) using the same frequency band at a 19.5 GHz carrier. Mounted on the antenna tower were one receiving horn antenna, which was dedicated to the terrestrial link, and a satellite dish emulating the downlink very small aperture terminal (VSAT) antenna that is interfered by signals transmitted from the prototype antenna array in the laboratory building. The angular separation of the terrestrial forward and satellite downlink was only about $1.5^{\circ}$. Despite such a small link separation and as aforementioned, the antenna array could steer one beam
SANSA deploys antenna arrays at the backhaul nodes to enable topology reconfiguration and frequency

reuse. At mmWave, a large number of antenna elements are required to combat path loss. Therefore,

given the cost performance trade-off, we opt for a hybrid analog-digital architecture.

toward the horn antenna and one null toward the satellite dish antenna by means of the hybrid beamforming. The outcome of the experiment was that the main beam established the terrestrial link, whereas the null successfully protected the satellite link, which could not be established when the antenna array emulated an antenna without null steering capabilities.

\section{Demonstration of the Hybrid Network Manager}

The second demonstration focused on the performance evaluation of the hybrid terrestrial-satellite flexible backhaul network by including the core functionalities of the HNM and iBNs PoCs.

As depicted in Fig. 5, the demonstration setup consisted of six base stations (BS1-BS6). Each BS was equipped with one iBN (iBN1-iBN6) featuring the number of virtual RF interfaces (i.e., terrestrial modems in a real deployment) indicated in brackets. The backhaul links were emulated at the network layer by means of a virtual LAN (VLAN) switch. Selected BSs were equipped with different access technologies (LTE eNB at BS5, wireless local loop at BS9, and WiFi access point at BS3) and users generating traffic, thus extending the SANSA solution beyond LTE. The hybrid (terrestrial-satellite) BS4 hosted an evolved packet core (EPC), an HNM, and a satellite modem. This BS was directly connected to the Internet. BS3 was also a hybrid BS that was equipped with a satellite modem. The two satellite modems were connected in a back-to-back fashion. Therefore, the multiple terrestrial links as well as one satellite backhaul link were realistically emulated in a mixed virtual and physical setup. This network was used to eval- 


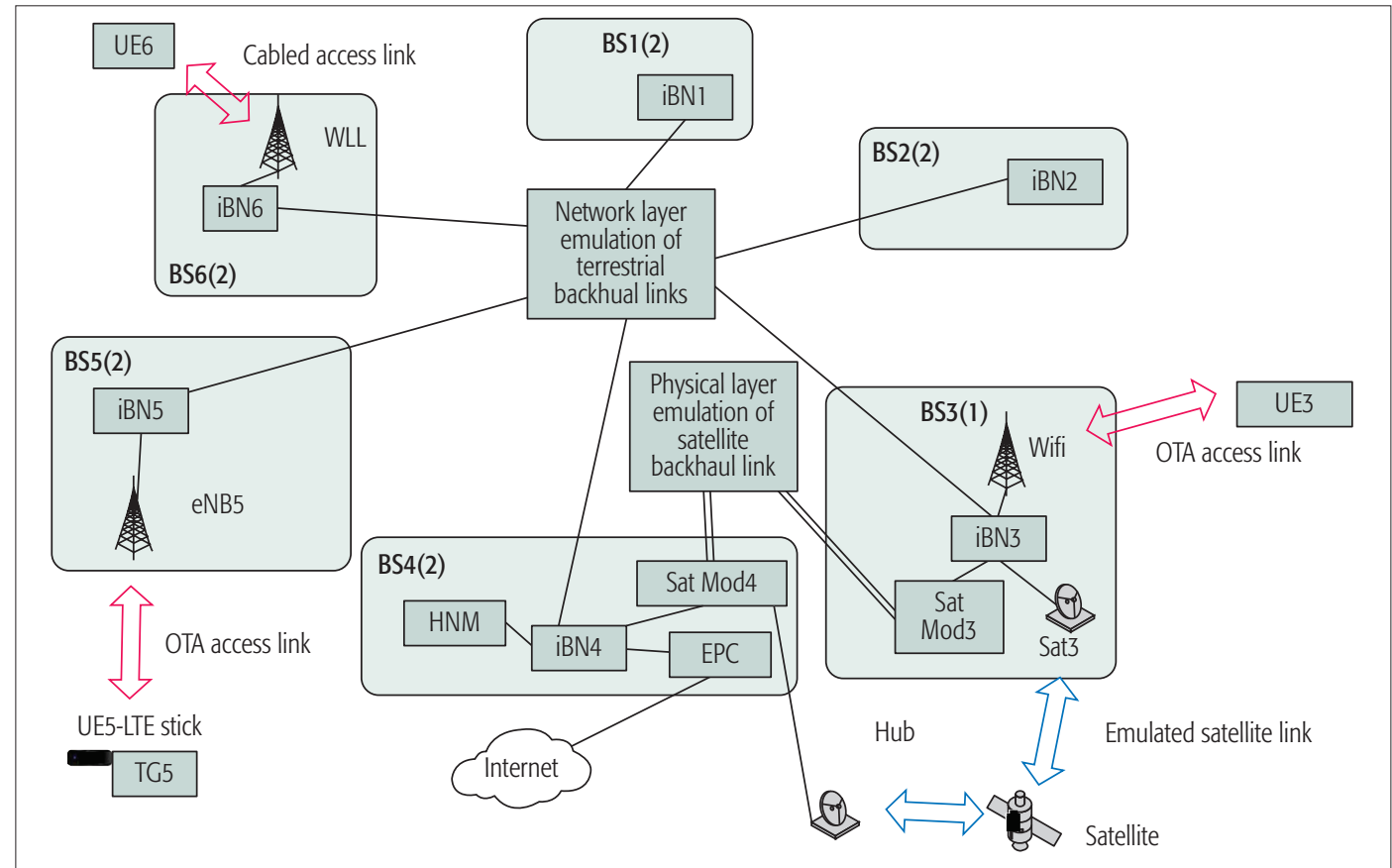

FIGURE 5. Demonstration setup for evaluation of the HNM performance and its ability to dynamically improve the backhaul network capacity and resilience.

This article presents and validates a novel hybrid management scheme for backhaul networks,

which enables the integration of the satellite segment in a topology-reconfigurable terrestrial wireless

network, resulting in a flexible solution capable of addressing $5 G$ challenges in both sparse and

dense network deployments.

uate the basic functionalities of the HNM and iBN PoCs. Their main role was to monitor the integrity of the backhaul network, to identify networking problems (link failure, congestion), and to propose a new topology with improved capacity. The obtained results confirmed the proper functionality of the HNM/iBNs combination. The link failures could be detected, and new topologies were proposed by the HNM and established by the iBNs, which improved the capacity and resilience of the network in comparison to traditional inflexible backhaul networks that are usually realized by means of fixed high directional antennas without any possibility to automatically adapt the network to the instantaneous link status.

\section{CONCLUSIONS AND Open ReSEARCH ChallengeS}

This article presents and validates a novel hybrid management scheme for backhaul networks, which enables the integration of the satellite segment in a topology-reconfigurable terrestrial wireless network, resulting in a flexible solution capable of addressing 5G challenges in both sparse and dense network deployments. Moreover, such integration is done under strong spectrum efficiency constraints, thus considering spectrum coexistence between terrestrial and satellite segments as well as aggressive frequency reuse between terrestrial wireless links. Both are enabled by dynamic RRM and HAD beamforming techniques.

There are still some open research challenges to be solved for bringing such a solution to reality. First, despite the proposed management scheme being based on pure software functions that could easily be aligned with the software defined networking and network functions virtualization 5G concepts, it is important to deal with the specific integration of those functions in the 5G network management framework. Second, deep integration of the topology manager and the radio resource manager functions is required for implementing an adaptive spectrum-efficient solution. Finally, although not addressed in the article due to space constraints, the experimental tests showed that the calibration of large hybrid analog-digital arrays is still complex and of vital importance in order to efficiently mitigate interference.

\section{ACKNOWLEDGMENT}

This work has received funding from the European Union's Horizon 2020 research and innovation programme under grant agreement No. 645047 (SANSA).

\section{REFERENCES}

[1] 5G Infrastructure Assn. white paper, "5G Vision"; https://5gppp.eu/wp-content/uploads/2015/02/5G-Vision-Brochurev1.pdf.

[2] NetWorld2020's SatCom WG, "The Role of Satellites in 5G," white paper, July 2014.

[3] S. Maleki et al., "Cognitive Spectrum Utilization in Ka Band Multibeam Satellite Communications," IEEE Wireless Commun., vol. 53, no. 3, June 2015, pp. 24-29.

[4] SANSA project ( H2020-ICT6-2014); http://sansa-h2020.eu/

[5] F. Mendoza, R. Ferrs, and O. Sallent, "A Traffic Distribution Scheme for 5G Resilient Backhauling Using Integrated Satellite Networks," Proc. 2017 13th Int'l. Wireless Commun. and Mobile Computing Conf., Valencia, Spain, 2017, pp. $1671-76$.

[6] R. Santos and A. Kassler, "Small Cell Wireless Backhaul Reconfiguration Using Software-Defined Networking," Proc. 2017 IEEE Wireless Commun. Networking Conf., San Francisco, CA, 2017, pp. 1-6.

[7] M. Vázquez, L. Blanco, and A. I. Pérez-Neira, "Hybrid AnalogDigital Transmit Beamforming for Spectrum Sharing Backhaul Networks," IEEE Trans. Signal Processing, vol. 66, no. 9, May 1, 2018, pp. 2273-85. 
[8] SANSA Project deliverable, "D4.3: Hybrid Network Management Design and Simulation Campaigns," July 2017; http:// sansa-h2020.eu/deliverables.

[9] SANSA Project deliverable, "D4.4: Multicast Beamforming for Distribution of Popular Multimedia Content towards the Terrestrial Distribution Network," July 2017; http://sansa-h2020.eu/deliverables.

[10] J. Baranda, J. Nez-Martnez, and J. Mangues-Bafalluy, "BPMR: Bakpressure Routing for the Heterogeneous Multi-Radio Bakhaul of Small Cells," Proc. 8th IFIP Wireless and Mobile Networking Conf., Munich, Germany, 2015, pp. 48-55.

[11] M. J. Neely, "Stochastic Network Optimization with Application to Communication and Queueing Systems," Synthesis Lectures on Commun. Networks, vol. 3, no. 1, 2010, pp $1-211$.

[12] X. Artiga et al., "Spectrum Sharing in Hybrid Terrestrial-Satellite Backhaul Networks in the Ka Band," Proc. 2017 Euro. Conf. Networks Commun., Oulu, Finland, 2017, pp. 1-5.

[13] E. Lagunas et al., "Resource Allocation for Cognitive Satellite Communications with Incumbent Terrestrial Networks," IEEE Trans. Cognitive Commun. Networking, vol. 1, no. 3, Nov. 2015, pp. 305-17.

[14] M. Shaat and A. I. Pérez-Neira, "Joint Flow Control and Link Scheduling in Hybrid Terrestrial-Satellite Wireless Backhauling Network," Proc. 2017 IEEE ICC Wksps., Paris, France, 2017, pp. 870-75.

[15] P. Gorski, M. C. Vigano, and D. Llorens del Rio, "Developments on Phased Array for Low-Cost, High Frequency Applications," Proc. 2017 11th Euro. Conf. Antennas and Propagation, Paris, France, 2017, pp. 436-38.

\section{BIOGRAPHIES}

XAVIER ARTIGA obtained his Ingeniero Superior degree in telecommunications (equivalent to an M.Sc.) at Universitat Politécnica de Catalunya (UPC) in July 2006. Since September 2007, he has worked at Centre Tecnologic de Telecomunicacions de Catalunya (CTTC/CETCA) as a researcher. He has participated in several national and European funded projects such as FP7-BuNGee and H2020-SANSA. His current research interests include reflect array and metasurface antennas, multi-antenna and massive MIMO systems, and radio frequency transceivers.

ANA I. PÉREZ-NeIRA is a full professor at UPC in the Signal Theory and Communication Department. Her research topic is signal processing for communications. She was Vice Rector for Research at UPC (2010-2013). She created the UPC Doctoral School (2011). Currently, she is a scientific coordinator at CTTC. From 2008 to 2016 she was a member of the EURASIP Board of Directors; from 2010 to 2016 she was a member of IEEE Signal Processing Theory and Methods, and in 2016 she was elected SPS Regional Director-at-Large for two years. She is the coordinator of the European project SANSA. She has been the leader of 20 projects and has participated in over 50 . She is an author of 50 journal papers and more than 200 conference papers. She is a recipient of the 2018 EURASIP Society Award.

JORGE BARANDa is currently a researcher in the Mobile Networks Department at CTTC, Barcelona. He received an M.Sc. (2008) degree in telecommunications engineering from UPC. At CTTC, he participates in national, European, and industrial projects related to the development of novel wireless communication systems and 5G mobile networks. His current research interests include wireless communications, wireless backhaul, network optimization, software-defined networking, and network functions virtualization.

EVA LAGUNAS [S'09, M'13, SM'18] received her M.Sc. and Ph.D. degrees in telecom engineering from UPC in 2010 and 2014, respectively. She was a research assistant within the Department of Signal Theory and Communications, UPC, from 2009 to 2013. She held visiting research appointments at the Department of Information Engineering, University of Pisa, Italy, in 2009, and at the Center for Advanced Communications (CAC), Villanova University, Pennsylvania, in 2011. Since 2014, she has been a research associate at the Interdisciplinary Centre for Security, Reliability and Trust (SnT), University of Luxembourg.

SYMEON ChATZINOTAS [S'06, M'09, SM'13] is currently the deputy head of the SIGCOM Research Group, SnT, University of Luxembourg, and a visiting professor at the University of Parma, Italy. He has over 300 publications, 3000 citations, and an H-Index of 28 according to Google Scholar. He was a co-recipient of the 2014 IEEE Distinguished Contributions to Satellite Communications Award, the CROWNCOM 2015 Best Paper Award, and the 2018 EURASIC JWCN Best Paper Award.
Koice, Slovakia. There, he also received a Ph.D. in 2001 on applications of time-frequency representations in UWB radar systems. In 2002, he joined the Electronic Measurement Research Lab at IImenau University of Technology, Germany. There, he habilitated in the area of UWB sensors for surveillance applications in emergency and security situations. Since 2014, he has been with Fraunhofer Institute for Integrated Circuits IIS, Germany. He became a team leader of the Radar, Channel Sounding and mmWave group. His research interests include UWB sensors, through-wall radars, real-time MIMO channel sounding, localization and imaging in UWB sensor networks, calibration of antenna arrays, hybrid (analog-digital) beamforming, localization services in cellular communication networks, and so on.

PRZEMYSLAW GORSKI received his M.Sc. and Ph.D. degrees in electronics from Wroclaw University of Technology, and is currently employed by Viasat Antenna Systems in the research and development department located in Lausanne, Switzerland. His current research interests lie in the area of phased array design and analysis.

KONSTANTINOS NTOUGIAS received his M.Sc. degree in electrical engineering from Darmstadt University of Applied Sciences in 2013. He is currently a Ph.D. candidate at Aalborg University (AAU) and a research engineer with Athens Information Technology (AIT). His research interests lie in the general field of communication theory, with main focus on MIMO communication, resources allocation, spectrum sharing, and caching techniques.

DAVID PÉREZ is currently working as a telecommunication engineer in the Software and Ground Department at Thales Alenia Space, Madrid. Licensed in Telecommunications and Electronic Engineering, in 2016, he received an M.Sc. in telecommunications Engineering at Staffordshire University, Stoke-on-Trent, United Kingdom. At Thales Alenia Space, he has participated in different European projects inside the FP7 (SESAR, GAMMA) and H2020 (SANSA, 5G-Ensure) frameworks. Also, he has made different contributions to internal products launched at the national and European level in terms of network management systems, software design and development, virtualization, 5G networks, wireless and hybrid networks, and different space matters.

GEORGIOS ZIARAGKAS works as a satellite communications engineer and project manager for Avanti Communications. His scientific interests are ground segment technologies for different services and applications as well as business modeling for innovative technological projects. He has also worked as a satellite ground station engineer. He holds an M.Eng. in electrical and computer engineering from Aristotle University of Thessaloniki and an M.B.A. from Athens University of Economics and Business. 